2016 - Volume: 17 Number: 5

Page: 905 - 914

DOI : $10.18038 /$ aubtda. 279858

Received: 08 September 2016 Revised: 21 November 2016 Accepted: 28 November 2016

\title{
EFFECT OF SYNTHESIS PARAMETERS ON ISONIAZID (INH) RELEASE FROM SILICA NANOSPHERES
}

\author{
Nagihan KARAKAYA ${ }^{1}$, Levent DEĞİRMENCI ${ }^{2,}$ * \\ ${ }^{1}$ Chemical and Process Engineering, Bilecik Şeyh Edebali University
}

\begin{abstract}
Silica nanospheres containing isoniazid (INH) was synthesized based on a modified Stober process. Synthesis parameters such as type of alcohol used as oil phase, tetra ethyl ortho silicate (TEOS) and ammonia $\left(\mathrm{NH}_{3}\right)$ amounts, surfactant use and mixing time were altered and their effect on the release of INH was investigated. Results indicated lower amount of INH release when higher amount of TEOS was utilized in synthesis. Time of highest INH release was higher in the case of higher TEOS amount. $\mathrm{NH}_{3}$ had also a decreasing effect on INH release which had been due to the changes in pore structure. Scanning electron microscope (SEM) results clearly showed the necessity of surfactant use with smooth surfaces obtained with the samples where surfactant was utilized. Finally, mixing time was found to have a decreasing effect on the time of highest INH release and the amount of INH released from the spheres was also higher in the case of higher mixing time
\end{abstract}

Keywords: Isoniazid, Nanosphere, Silica, Stober process

\section{INTRODUCTION}

Polymeric nanoparticles, due to various synthesis procedures developed so far, have a great variety of applications in electronics, biotechnology, environmental technology and pollution control [1]. These materials are favored in controlled release of various drugs for their ability to carry and deliver the active material situated inside their structure [2]. Anti-cancer drugs [2,3], calcium channel blocker [4] and antibiotics designed to cure tuberculosis [5] are among many examples.

Investigations on the potential of silica based mesoporous structures have increased since first utilization of MCM-41 as drug career [6]. Biomedical applications require a precise control on physical characteristics of the synthesized material. Silica based materials steps in with their homogeneous pore size distribution, high pore volume and high surface area [6,7].

Tuberculosis infection is among the most important disease which tend to develop especially in children $[8,9]$. Certain treatments are being developed for the disease, yet the drugs used in its cure cause drug induced liver injury which is more likely to occur in people with an alcohol background [10]. Liver injury is the result of the challenges in detoxification of the drug and the possibility of injury increases with the amount of drug uptake [10]. In order to prevent drug induced liver injury during treatment, perfect control of drug dosage taken by the liver must be maintained. Controlled release of the drug to the body serve as an excellent solution for the problem. Isoniazid is known as the most effective drug used in tuberculosis treatment [11] and since it is mostly detoxified in the liver [10], controlled release of this material is very important to prevent liver injury and maintain an effective treatment at the same time.

Microencapsulation is an effective method in controlled release of isoniazid. Silica based nanospheres are popular among the researchers as this compound is chemically inert, has high mechanical and thermal stability and it is easy to incorporate various groups to enhance release of the material inside

*Corresponding Author: levent.degirmenci@bilecik.edu.tr 
[12]. Silica nanospheres are usually synthesized by a well-known Stober process. Particle size of the nanospheres obtained during synthesis can be altered by simple modifications such as changing the solvent ratio, silica source and the surfactant [13-15].

Silica based mesoporous materials had recently been synthesized in hollow, and solid type core mesoporous shell form [16]. Applications include loading of carvedilol [17], ibuprofen [18], felodipine [19] and avermectin [20]. Tetraethyl ortho silicate (TEOS) $[17,19,20]$ and sodium silicate [18] were utilized as silica sources and microspheres were synthesized in the presence of various surface active materials such as cethyl tri methyl ammonium bromide (CTAB), Tween 20 and Span 80.

In the present study silica based isoniazid (INH) containing nanospheres were synthesized based on Stober process $[21,22]$. Synthesis procedures used in the study have been modified by changing the amount of TEOS and ammonia $\left(\mathrm{NH}_{3}\right)$, the type of alcohol used as the oil phase and mixing time of the synthesis solution in order to determine their effect on release characteristics of INH.

\section{MATERIAL AND METHODS}

\subsection{Synthesis of Silica Nanospheres}

Preparation of a water/oil emulsion to achieve sphere formation during consecutive hydrolysis and condensation of TEOS was the first step in synthesis. Water phase was prepared by dissolving INH in deionized water. Oil phase consisted of a short chain alcohol and $25 \% \mathrm{NH}_{3}$ solution, which was emulsified in oil phase by homogenization for 5 minutes. Tween 80 was dispersed in oil phase along with $\mathrm{NH}_{3}$ by homogenization when utilized.

Samples were denoted as K1, K2, U1, U2, U3, U4, U5 and U6. K code was given for the spheres synthesized in the presence of $48 \mathrm{ml}$ of 2-propanol in the oil phase and $\mathrm{U}$ code was given for the spheres synthesized in the presence of $50 \mathrm{ml}$ of ethanol used in the oil phase. A typical procedure for the synthesis of $\mathrm{K} 1$ is as follows:

- $0.5 \mathrm{~g}$ INH was dissolved in $20 \mathrm{ml}$ of deionized water by ultrasonification (water phase).

- Oil phase $(\mathrm{O})$ was prepared by mixing $60 \mathrm{ml}$ of $\mathrm{NH}_{3}$ and $48 \mathrm{ml}$ of 2-propanol in a homogenizer (5000 rpm 5 minutes).

- Water phase was added to oil phase.

- The resulting solution was mixed in a propeller at $300 \mathrm{rpm}$ for 6 hours and $5 \mathrm{ml}$ of TEOS was added dropwise to this solution.

- Nanospheres were removed from the solution by centrifuging for 5 minutes at $4000 \mathrm{rpm}$. Removed samples were then washed with ethanol and centrifuged again. A total of 3 washing/centrifuging cycles were applied to remove impurities.

The amount of TEOS added to the solution was determined based on weight ratio of TEOS/INH which was determined as 10 in the course of studies. A summary of the applied procedures and the changes conducted in synthesis conditions were illustrated in Table 1, to avoid confusion and provide a better follow-up. 
Table 1. Summary of applied procedures in the present study

\begin{tabular}{lllll}
\hline Code & $\begin{array}{l}\text { Water phase* } \\
(\mathbf{g})\end{array}$ & $\begin{array}{l}\text { Oil phase** } \\
(\mathbf{m l})\end{array}$ & $\begin{array}{l}\text { Tween 80 } \\
(\mathbf{g})\end{array}$ & Mixing time (h) \\
$\mathrm{K} 1$ & 0.5 & 60 & - & 6 \\
$\mathrm{~K} 2$ & 0.5 & 60 & 0.25 & 6 \\
$\mathrm{U} 1$ & 0.3 & 10 & - & 4 \\
$\mathrm{U} 2$ & 0.3 & 10 & 0.25 & 4 \\
$\mathrm{U} 3$ & 0.3 & 20 & 0.25 & 4 \\
$\mathrm{U} 4$ & 0.3 & 60 & 0.25 & 4 \\
U5 & 0.5 & 10 & 0.25 & 4 \\
U6 & 0.5 & 10 & 0.25 & 6 \\
* Water phase was prepared by dissolving designated amounts of $\mathrm{INH}$ in 20 ml deionized water. \\
**Amount of $\mathrm{NH}_{3}$ added to 48 ml of 2-propanol (K1 and K2) and 50 ml of ethanol
\end{tabular}

\subsection{Characterization Studies}

Scanning Electron Microscope (SEM) and Nitrogen $\left(\mathrm{N}_{2}\right)$ physisorption analyses were conducted on nanospheres to determine the effect of applied synthesis procedure on sphere formation and pore size distribution.

\subsection{In Vitro Release Studies}

Controlled release of INH from silica nanospheres was conducted in a Phosphate-buffared saline (PBS) solution prepared by dissolving PBS in $200 \mathrm{ml}$ pure water and the $\mathrm{pH}$ of the solution was held constant at 7.4 to imitate the conditions of human blood. Release medium was prepared by placing 50 $\mathrm{mg}$ of nanosphere in $20 \mathrm{ml}$ of PBS solution. Samples were kept in a shaking incubator at $37^{\circ} \mathrm{C}$ with a constant agitation of $100 \mathrm{rpm}$. In vitro release studies were conducted with samples taken and analyzed hourly for the first 8 hours and then 12 hours interval for 3 days. A total of 9 solutions with identical release medium were utilized in analyses. The samples were centrifuged at $4000 \mathrm{rpm}$ for 5 minutes to remove nanospheres and the remaining fluid was poured in a test tube and absorbance values of the fluid were obtained via ultra violet (UV) spectrophotometer at the wavelength of 262 nm.

\section{RESULTS AND DISCUSSION}

SEM analyses of the samples (Figure 1 and 2) indicated cracks on the surface and deformation on sphere formation of K1 and U1 samples implying the necessity of surfactant utilization to obtain smooth surfaces. Deformation of sphere formation and surface morphology are among the most important problems in drug release since deviations from sphere formation increase the friability of the structure resulting in uncontrolled release of the drug inside the structure [23]. Another morphology related problem in nanospheres is the surface roughness which revealed itself as cracks in $\mathrm{K} 1$ and U1 samples. Smooth surfaces are needed to prevent fast release of the drug which was also identified as burst release in the work of Huang et.al. [24]. Rough surfaces accelerate burst release of the drug and most of the drug is released uncontrollably during the process [24]. Consequently, obtaining smooth surfaces is a necessity which make utilization of surfactant mandatory. Effect of INH and $\mathrm{NH}_{3}$ amount and mixing time of synthesis solution had no significant effect on sphere formation (Figure 1 and 2). 
Karakaya and Değirmenci / Anadolu Univ. J. of Sci. and Technology-A-Appl. Sci. and Eng. 17 (5) - 2016
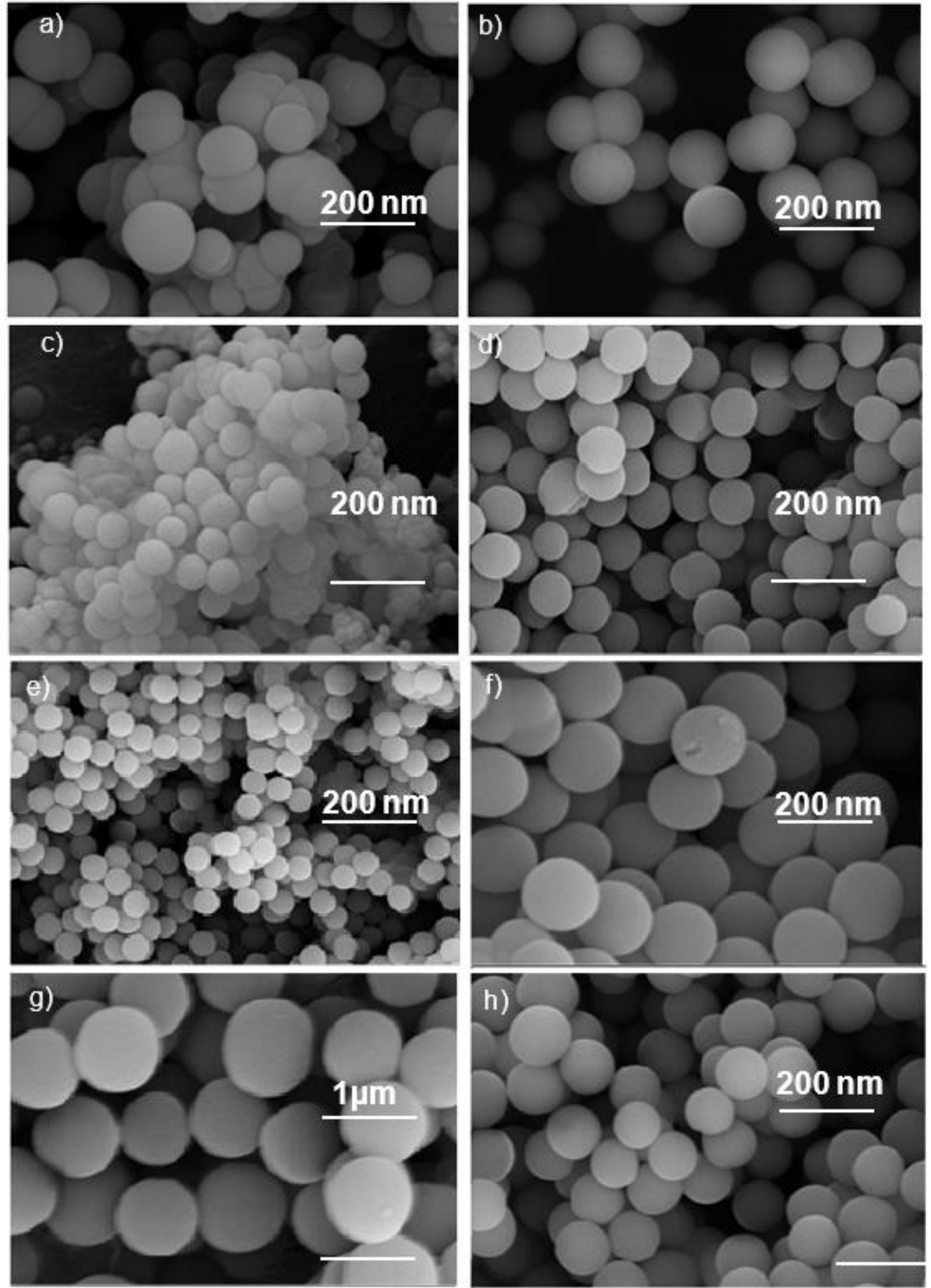

Figure 1. SEM images of a) K1 b) K2 c) U1 d) U2 e) U3 f) U4 g) U5 and h) U6 nanospheres 

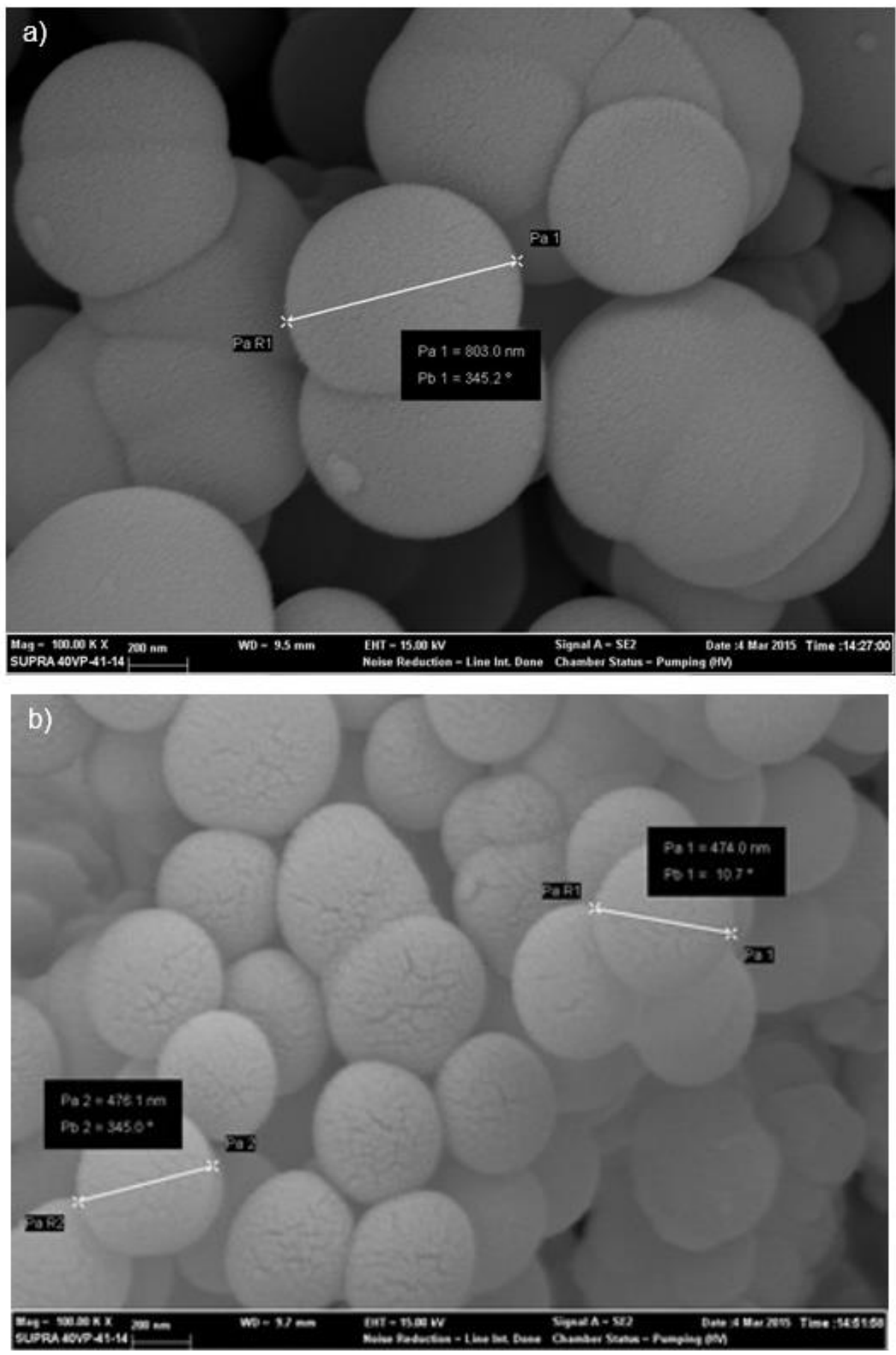

Figure 2. SEM images of a) K1 b) U1 nanospheres

Physisorption analyses were conducted to determine $\mathrm{N}_{2}$ adsorption-desorption isotherms of K2, U2, U3, U4, U5 and U6 samples. Since the isotherms of U2, U3, U4, U5 and U6 were similar, only the isotherms of $\mathrm{K} 2$ and $\mathrm{U} 2$ were illustrated in Figure 3 to maintain easy follow up of the results. Physisorption analysis report of the nanospheres were given in the supplementary file. $\mathrm{N}_{2}$ adsorptiondesorption isotherms of $\mathrm{K} 2$ and $\mathrm{U} 2$ nanospheres indicated a type IV isotherm with H3 hystresis loop indicating a mesoporous structure. This type of isotherm is observed when a monolayer of adsorbate is initially formed on the pore surface followed by multilayer formation. The obtained isotherm also implied distribution of isoniazid throughout the sphere. However, a negligible increase of volume has 
been observed for both nanospheres with relative pressure values between 0.3-0.8. This result implied low quality of the mesopores inside the spheres [25]. Adsorption-desorption isotherms and pore size distribution of K2 and U2 revealed identical structures (Figure 3). Overall, physisorption results showed formation of similar mesoporous structures and that the type of oil phase utilized in syntheses had not been effective in pore structure. Physical properties of the samples obtained from physisorption analyses were illustrated in Table 2. Amount of $\mathrm{NH}_{3}$ added to synthesis solution resulted in formation of smaller nanospheres with larger pore volumes and surface area. As mentioned in Table 1, 2-propanol was utilized in the oil phase in the synthesis of $\mathrm{K} 2$ nanospheres. Other than the type of the alcohol used in the oil phase, the synthesis conditions of K2 and U4 were similar. Hence the results obtained with the nanospheres must be evaluated based on the type of alcohol used in the oil phase for $\mathrm{K} 2$ and $\mathrm{U} 4$ and the amount of $\mathrm{NH}_{3}$ used as the catalyst for the consecutive hydrolysis and condensation of TEOS in the case of U2- U6 nanospheres. The surface area and pore volume of $\mathrm{U} 4$ was higher than $\mathrm{K} 2$ and nanoparticle size of $\mathrm{U} 4$ was lower than $\mathrm{K} 2$ sample. This result indicated that ethanol was a better choice as a solvent than 2-propanol in synthesis. Amount of $\mathrm{NH}_{3}$ was another parameter to be evaluated in synthesis and results indicated formation of a smaller nanosphere with higher surface area and larger pores when $\mathrm{NH}_{3}$ amount was increased in synthesis solution.
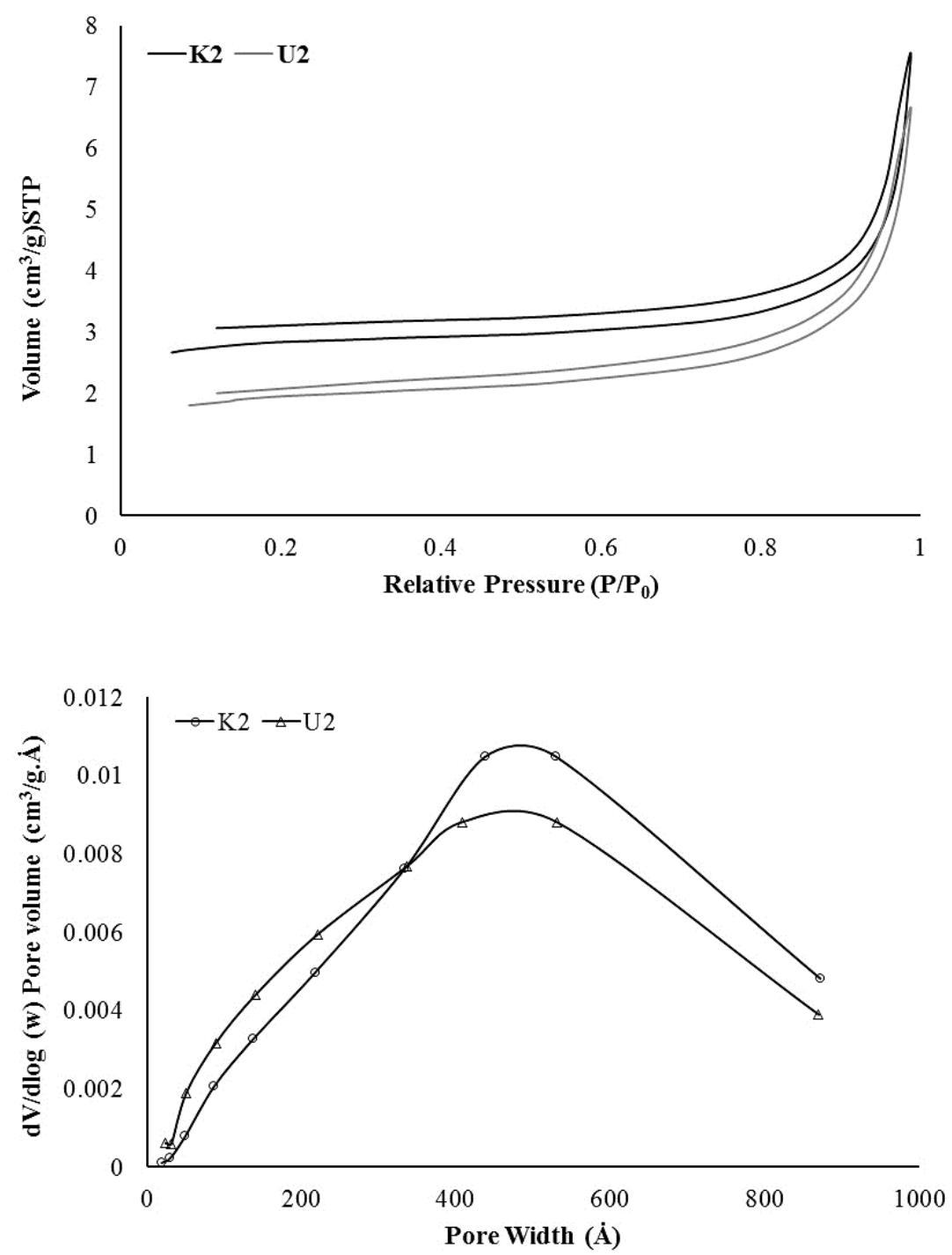

Figure 3. a) $\mathrm{N}_{2}$ adsorption-desorption isotherms b) Pore size distribution of K2, U2 nanospheres 
Table 2. Physical properties of silica nanospheres

\begin{tabular}{lllll}
\hline Sample & BET surface area $\left(\mathbf{m}^{\mathbf{2}} / \mathbf{g}\right)$ & Pore volume $\left(\mathbf{c m}^{\mathbf{3}} \mathbf{g}\right)$ & Pore size $(\mathbf{n m})$ & $\begin{array}{l}\text { Nanoparticle } \\
(\mathbf{n m})\end{array}$ \\
K2 & 8.0 & 0.008 & 5.34 & size \\
U2 & 6.2 & 0.008 & 6.22 & 963 \\
U3 & 8.9 & 0.012 & 6.47 & 677 \\
U4 & 12.1 & 0.017 & 6.65 & 495 \\
U5 & 10.6 & 0.012 & 5.74 & 566 \\
U6 & 6.7 & 0.009 & 6.70 & 892 \\
\hline
\end{tabular}

In vitro release studies obtained with $\mathrm{K} 2, \mathrm{U} 2, \mathrm{U} 3, \mathrm{U} 4, \mathrm{U} 5$ and U6 nanospheres were illustrated in Figures 4 and 5, respectively. Results indicated a rapid release of INH from nanosphere for U2 sample with release $\%$ reaching to 100 . Release of INH was higher than $90 \%$ at the $8^{\text {th }}$ hour with U2 nanospheres. Comparison of U2, U3 and U4 nanospheres indicated a decrease of INH release which was thought to be due to the amount of $\mathrm{NH}_{3}$ used in syntheses. Increase in $\mathrm{NH}_{3}$ amount resulted in the decrease of release \%. Effect of $\mathrm{NH}_{3}$ was mainly on surface area, pore volume and pore size of nanospheres which had elevated with $\mathrm{NH}_{3}$ surplus in synthesis solution. It was interesting to observe a decrease in released amount of INH although surface area and pore volume of nanospheres had risen. This decrease was thought to be due to the equilibrium between diffused amounts of PBS inside the pores of nanosphere and INH to PBS solution. It was thought that increase of pore volume had enhanced the amount of PBS diffused inside the nanosphere causing a decrease in released amount of INH (Figure 4). Similar conclusion could be reached with nanospheres with higher INH amounts (K2, U5 and U6) in which highest release rate had been obtained with K2 nanosphere (Figure 5). On the other hand, released amount of INH was lower in the case of high INH loading.

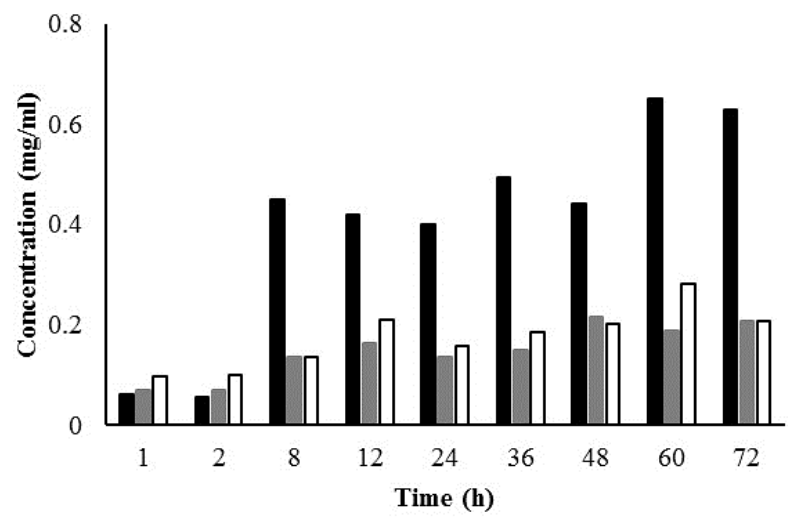

-U2 $=\mathrm{U} 3$ पU4

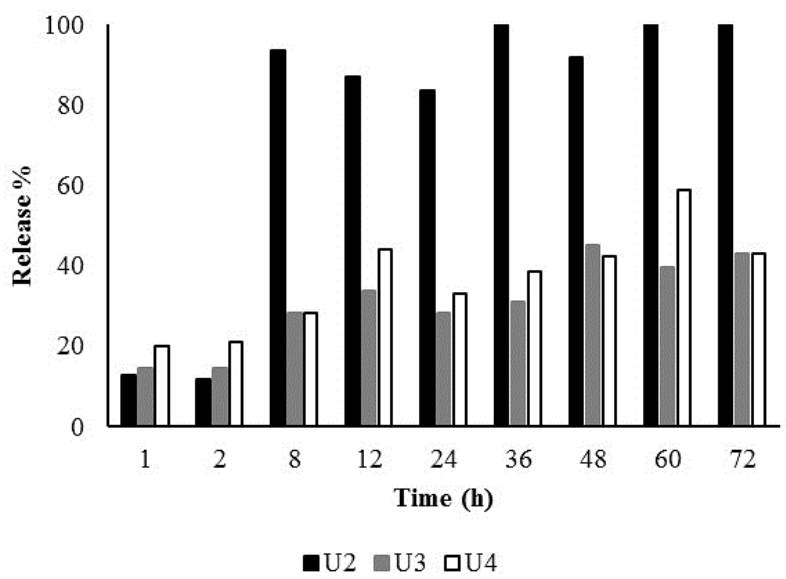

Figure 4. The change of a) INH concentration and b) release \% in PBS solution for nanospheres loaded with $0.3 \mathrm{~g} \mathrm{INH}$ 

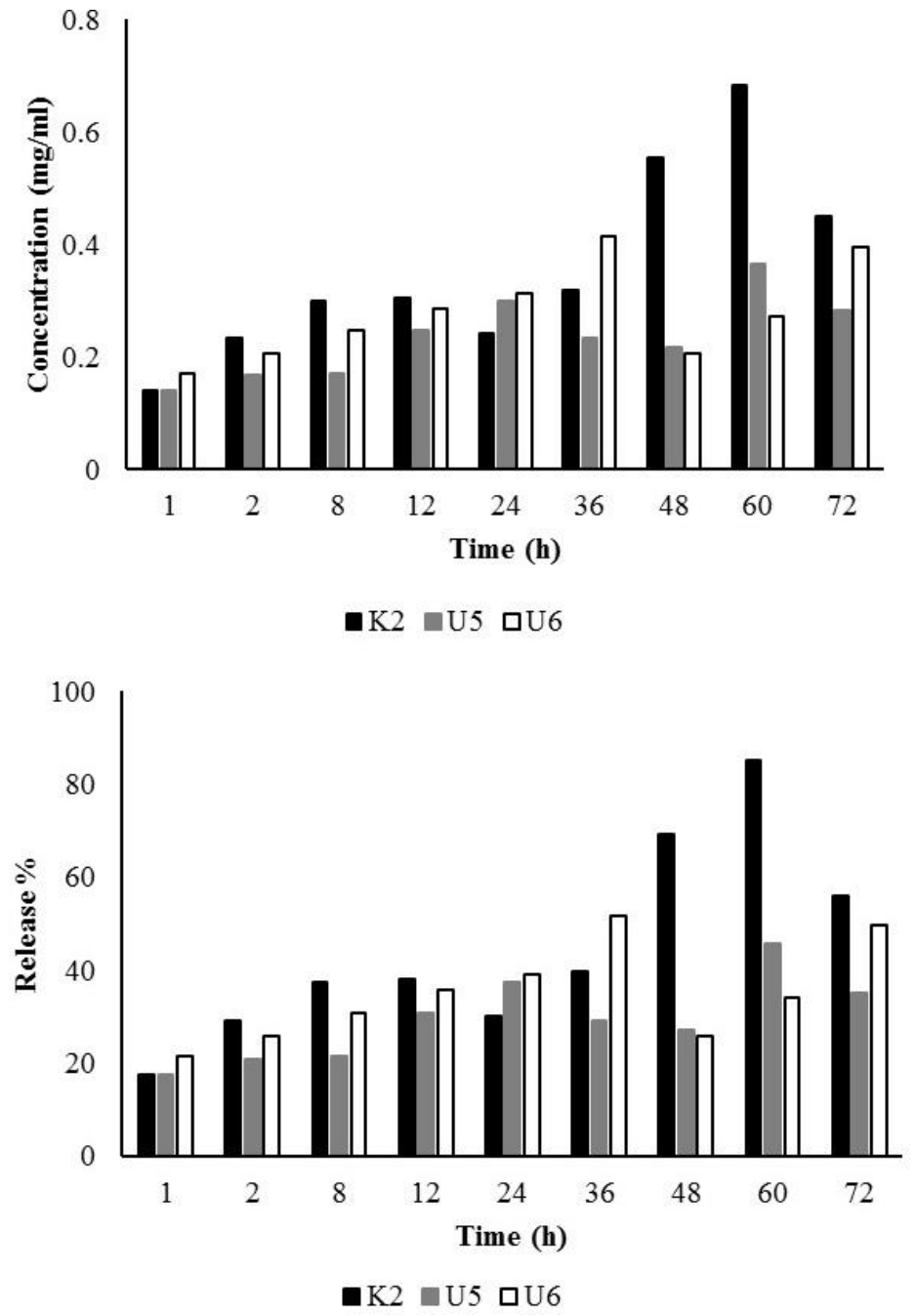

Figure 5. The change of a) INH concentration and b) release $\%$ in PBS solution for nanospheres loaded with $0.5 \mathrm{~g}$ INH

It is well known that hydrophilic polymers had the tendency to swell when immersed in water. Thus swelling of the nanosphere might increase with the amount of water introduced to the pores with PBS solution. Since higher pore volumes will contain higher amounts of PBS, higher swelling of the nanospheres with higher pore volumes must be expected. A diffusion layer formed as a result of swelling will restrict diffusion from the pores and maintain a controlled release environment. Results obtained with varying $\mathrm{NH}_{3}$ revealed that released amount of INH could have been controlled by simply adjusting the amount of $\mathrm{NH}_{3}$ utilized in synthesis [26].

\section{CONCLUSION}

Evaluation of release studies and physisorption analyses revealed the effect of varying synthesis parameters on INH release. Increase in $\mathrm{NH}_{3}$ amount resulted in the increase of surface area, pore volume and pore size; yet these changes reflected as a decrease in INH amount. This result was thought to be due to the equilibrium of PBS diffusion inside and outside the pores of the spheres. Swelling of silica structure is due to the PBS solution migrated inside the pores, the amount of which had increased with the surplus of pore volume. Hence a diffusion layer formed as a result of swelling resulted in controlled release of INH. Results obtained from release studies and physisorption analyses indicated the possibility of control on release time of INH by simply changing $\mathrm{NH}_{3}$ amount 
Karakaya and Değirmenci / Anadolu Univ. J. of Sci. and Technology - A-Appl. Sci. and Eng. 17 (5) - 2016

utilized in synthesis. Highest release rate was observed with U2 samples where lowest surface area, pore volume and pore size had been determined with lowest $\mathrm{NH}_{3}$ amount.

\section{REFERENCES}

[1] Rao JP, Geckeler KE. Polymer nanoparticles: Preparation techniques and size-control parameters. Prog Polym Sci 2011; 31: 887-913.

[2] Yoo HS, Oh JE, Lee KH, Park TG. Biodegradable Nanoparticles Conaining Doxorubicin-PLGA Conjugate for Sustained Release. Pharmaceut Res 1999;16 (7): 114-118.

[3] Albarran L, Lopez T, Ouintana P, Chagoya V. Controlled release of IFC-305 encapsulated in silica nanoparticles for liver cancer synthesized by sol-gel. Colloid A 2011;384: 131-136.

[4] Maver U, Godec A, Bele M, Planinsek O, Gaberscek S, Srcic S, Jamnik J. Novel hybrid silica xerogels for stabilization and controlled release of drug. Int J Pharm 2005; 330: 161-174.

[5] Xu W, Wei X, Wei K, Cao A. A mesoporous silicon/poly-(DL-lactic-co-glycolic) acid microsphere for long time anti-tuberculosis drug delivery. Int J Pharm 2014; 476: 116-123.

[6] Vallet-Regi M, Balas F, Arcos D. Mesoporous materials for durg delivery. Angew. Chem. Int. Ed 2007; 46: 7548-7558.

[7] Tang F, Li L, Chen D. Mesoporous silica nanoparticles: synthesis, biocompatibility and drug delivery. Advanced Materials 2012;24:1504-1534.

[8] Kontturi A, Santiago B, Tebruegge M, Both U, Salo E, Ritz N. The impact of Bacille CalmetteGuerin shortage on immunisation practice and policies in Europe- A Paediatric Tuberculosis Network European Trials Group (pbtnet) survey. Tuberculosis 2016; 101: 125-129.

[9] Singh G, Dwivedi H, Saraf S K, Saraf S A. Niosomal Delivery of Isoniazid- Development and Characterization. Tropical Journal of Pharmaceutical Research 2011; 10 (2): 203-210.

[10] Saukkonen JJ, Cohn D L, Jasmer RM, Schenker S, Jereb J A, Nolan C M, Peloquin C A, Gordin F M, Nunes D, Strader D B, Bernardo J, Venkataramanan R, Sterling T R. An official ATS Statement: Hepatotoxicity of antituberculosis therapy. Am J. Respir Crit Care Med 2006; 174: 935952.

[11] Verma R, Kumar L, Kurba VB. Isoniazid- A wonder drug in Tuberculosis management. Advances in Life Sciences and its Applications 2013; 1 (4): 98-103.

[12] Degirmenci L, Orbey N. Microencapsulation of silicotungstic acid to retain catalytic activity. Industrial \& Engineering Chemistry Research 2013; 52: 16714-16718.

[13] Venkatathri N, Synthesis of silica nanosphere from homogeneous and heterogeneous systems. Bull. Mater. Sci 2007; (30) 6: 615-617.

[14] Sato-Berru R, Saniger JM, Flores JF, Espindola M S. Simple method for the cotrolled growth of $\mathrm{SiO}_{2}$ spheres. Journal of Materials Science and Engineering A 2013; 3 (4): 237-242.

[15] Yun DS, Kim HY, Yoo JW. Preperation of silica nanospheres: effect of silicon alkoxide and alcohol on silica nanospheres. Bull. Korean Chem. Soc 2005; 26 (12): 1927-1928. 
[16] El-Toni AM, Khan A, Ibrahim MA, Labis JP, Badr G, Al-Hoshan M, Yin S, Sato T. Synthesis of double mesoporus core-shell silica spheres with tunable porosity and their drug release and cancer cell apoptosis properties. J Colloid Interf Sci 2012; 378: 83-92.

[17] Hu Y, Zhi Z, Zhao Q, Wu C, Zhao P, Jiang H, Jiang T, Wang S. 3D cubic mesoporous silica microsphere as a carrier for poorly soluble drug carvedilol. Micropor Mesopor Mat 2012; 147: 94101.

[18] Santamaria E, Maestro A, Porras M, Gutierrez JM, Gonzalez C. Controlled release of ibuprofen by meso-macroporus silica. J Solid State Chem 2014; 210: 242-250.

[19] Hu L, Sun H, Zhao Q. Multilayer encapsulated mesoporous silica nanospheres as an oral sustained drug delivery system for the poorly water-soluble drug felodipine. Mater Sci Eng 2015; 47: 313-324.

[20] Zhanga C, Houa T, Chena J, Wena L. Preparation of mesoporous silica microspheres with multihollow cores and their application in sustained drug release. Particuology. 2010; 8: 447-452.

[21] Ibrahim IAM, Zikry AAF, Sharaf MA. Preparation of spherical silica nanoparticles: Stober silica. J Am Sci 2010; 6 (11): 985-989.

[22] Gholami T, Slavati-Niasari M, Bazarginopour M, Noori E. (2013). Synthesis and characterization of spherical silica nanoparticles by modified Stöber process assisted by organic ligand. Superlattice Microst 2013; 61: 33-41.

[23] Wen H, Kinam P. Oral controlled formulation design and drug delivery. New Jersey, NY, USA: Wiley, 2010.

[24] Huang X, Brazel CS. On the importance and mechanisms of burst release in matrix-controlled drug delivery systems. Journal of Controlled Release 2001; 73: 121-126.

[25] Cui J, Sun H, Luo Z, Sun J, Wen Z. Preperation of low surface area $\mathrm{SiO}_{2}$ microsphere from wheat husk ash with a facile precipitation process. Mater Lett 2015; 156: 42-45.

[26] Rastogi R, Sultana Y, Aqil M, Ali A, Kumar S, Chuttani K, Mishra A K. Alginate microspheres of isoniazid for oral sustained drug delivery. International Journal of Pharmaceutics 2007; 334: 71-77. 